Classification

Physics Abstracts

61.16Di $-61.50 \mathrm{Jr}-82.65 \mathrm{Dp}$

\title{
Periodic faceting of Au(4,3,3) observed by scanning tunneling microscopy
}

\author{
Farhad Pourmir, Sylvie Rousset, Sébastien Gauthier, Micheline Sotto and Jean Klein
}

Groupe de Physique des Solides, Universités Paris 7 et 6, Laboratoire associé au C.N.R.S., 2 Place Jussieu, 75251 Paris, France

(Received July 4; accepted December 16, 1994)

\begin{abstract}
Résumé . - Une surface vicinale de l'or (1,1,1), Au(4,3,3), a été étudiée par microscopie à effet tunnel dans l'ultra vide. Elle est thermiquement instable et facette pour des températures de recuit allant de $700 \mathrm{~K}$ à $1000 \mathrm{~K}$. La surface facettée présente un état stable constitué d'une succession périodique de facettes ayant des orientations $(1,1,1)$ et $(7,5,5)$. La superpériode observée est d'environ $110 \AA$ avec une longueur de $40 \AA$ pour la facette dense et ne varie pas dans la gamme de température utilisée. Plusieurs origines pouvant rendre compte de la stabilité particulière de la surface $(7,5,5)$ et de la superpériode sont discutées.
\end{abstract}

\begin{abstract}
The $\mathrm{Au}(4,3,3)$ surface, vicinal to $\mathrm{Au}(1,1,1)$, has been studied by scanning tunneling microscopy in ultra high vacuum. It is thermally unstable towards faceting. For annealing temperatures ranging from $700 \mathrm{~K}$ to $1000 \mathrm{~K}$, the stable state of the faceted surface is found to be a periodic succession of $(1,1,1)$ and $(7,5,5)$ facets. The superperiod is about $110 \AA$ and the length of the close-packed facet is about $40 \AA$. The occurence of the $(7,5,5)$ facet is discussed in correlation with previous equilibrium shape observations. A model to qualitatively account for the periodic formation of facets is proposed.
\end{abstract}

Specifying the macroscopic orientation of a surface is not sufficient to know its morphology. Equilibrium morphology is indeed determined by the anisotropy of the surface free energy $\gamma$ [1]. A surface of macroscopic orientation $\phi$ can be unstable towards faceting: it minimizes the surface free energy by breaking up into a hill-and- valley structure. Such a faceting phenomenon appears upon thermal treatment or chemical adsorption. Due to its importance in equilibrium shape of crystals, catalyst, nucleation and growth, the study of faceting has been of great interest for many decades [2]. However how mass transport occurs during the faceting process is still a challenging question. Since real-space imaging techniques and better characterisation of the property of surfaces have become available, it is now possible to study faceting at the atomic scale level [3]. Scanning tunneling microscopy [4] provides a unique insight into this phenomenon since it reveals a direct view of the nucleation of facets and their spatial arrangement. It has been extensively used on silicon [5] and only a very few studies have been performed on metals [6]. In the case of gold the anisotropy of the surface free energy has been studied by two different techniques $[7,8]$ and it 
seems that the equilibrium shape of the crystal in the vicinity of the facets $((111)$ and $(100)$ orientations) presents sharp edges. According to the Herring construction [9], it significates that vicinal surfaces close to the close-packed surfaces (111) or (100) should be unstable towards faceting. We have investigated the thermal stability of a vicinal surface of $\mathrm{Au}(111)$, belonging to the [011] zone axis. Previous studies of thermal faceting on gold surfaces concern mainly vicinals of $\mathrm{Au}(100)$ [10] and vicinals of $\mathrm{Au}(111)$ in the [112] zone axis [11]. "Magic vicinals" have been deduced from these observations. They have been experimentally and theoretically explained by a relationship between their terrace width and the length of the unit cell of the well-known reconstructions [12] $(5 \times 1$ on $\mathrm{Au}(100)$ [13] and $22 \times \sqrt{3}$ on $\mathrm{Au}(111)$ [14]). However they have not been interpreted by a comparaison with the equilibrium shape of crystals.

The $\mathrm{Au}(4,3,3)$ surface is slightly misoriented from the (111) close-packed orientation by an angle of $8.05^{\circ}$. Ideally its stepped structure is represented figure 1 . It consists of a periodic succession of (111) terraces separated by $\{100\}$ microfacets. The width of the terraces is $16.6 \AA$ (i.e. 7 atomic rows) and the steps height is monoatomic, $h=2.35 \AA$ high. The step edges are aligned along the zone axis direction, [011]. The sample was cut by spark erosion, mechanically polished and finally etched. This ex-situ preparation was done elsewhere [15]. Once introduced inside the ultra high vacuum chamber, which operating pressure is in the low $10^{-10}$ Torr range, the sample is preparared in-situ by cycles of $\mathrm{Ar}^{+}$ion bombardment $\left(400 \mathrm{eV}, 5 \times 10^{-6} \mathrm{Torr}, 5 \mathrm{~min}\right)$ and annealing. Temperatures of annealing reported here range from $700 \mathrm{~K}$ to $1000 \mathrm{~K}$. At higher temperatures the calcium, contained in the bulk as an impurity, could segregate towards the surface. The sample is allowed to cool about 2 hours before it is observed with a home-built scanning tunneling microscope (STM) described in detail previously [16]. Tungsten tips are etched by electrochemical erosion and cleaned in-situ by resistive heating up to about $1000^{\circ} \mathrm{C}$ before experiments. The conditions of tunneling current and sample tunneling voltage are respectively $1 \mathrm{nA}$ and a few hundred $\mathrm{mV}$ negative.

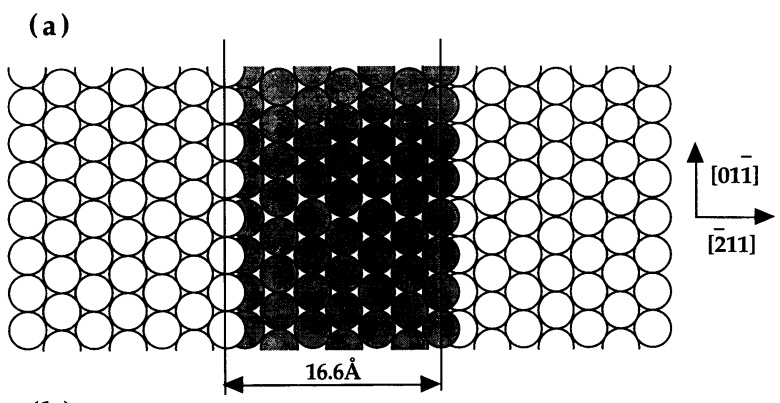

(b)

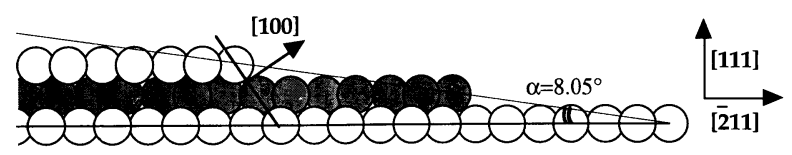

Fig. 1. - Ideal structure of the $\mathrm{Au}(4,3,3)$ surface from a hard-sphere model; a) top view, b) side view.

When the annealing temperature is above $700 \mathrm{~K}$, the $\mathrm{Au}(4,3,3)$ surface breaks up into a hilland-valley structure. STM images obtained on the faceted surface are presented in figures 2 and 3. Large scale images reveal the long range order of the surface (cf. Fig. 2) whereas better resolved images characterize the hill-and-valley structure (cf. Fig. 3). Succession of close-packed 


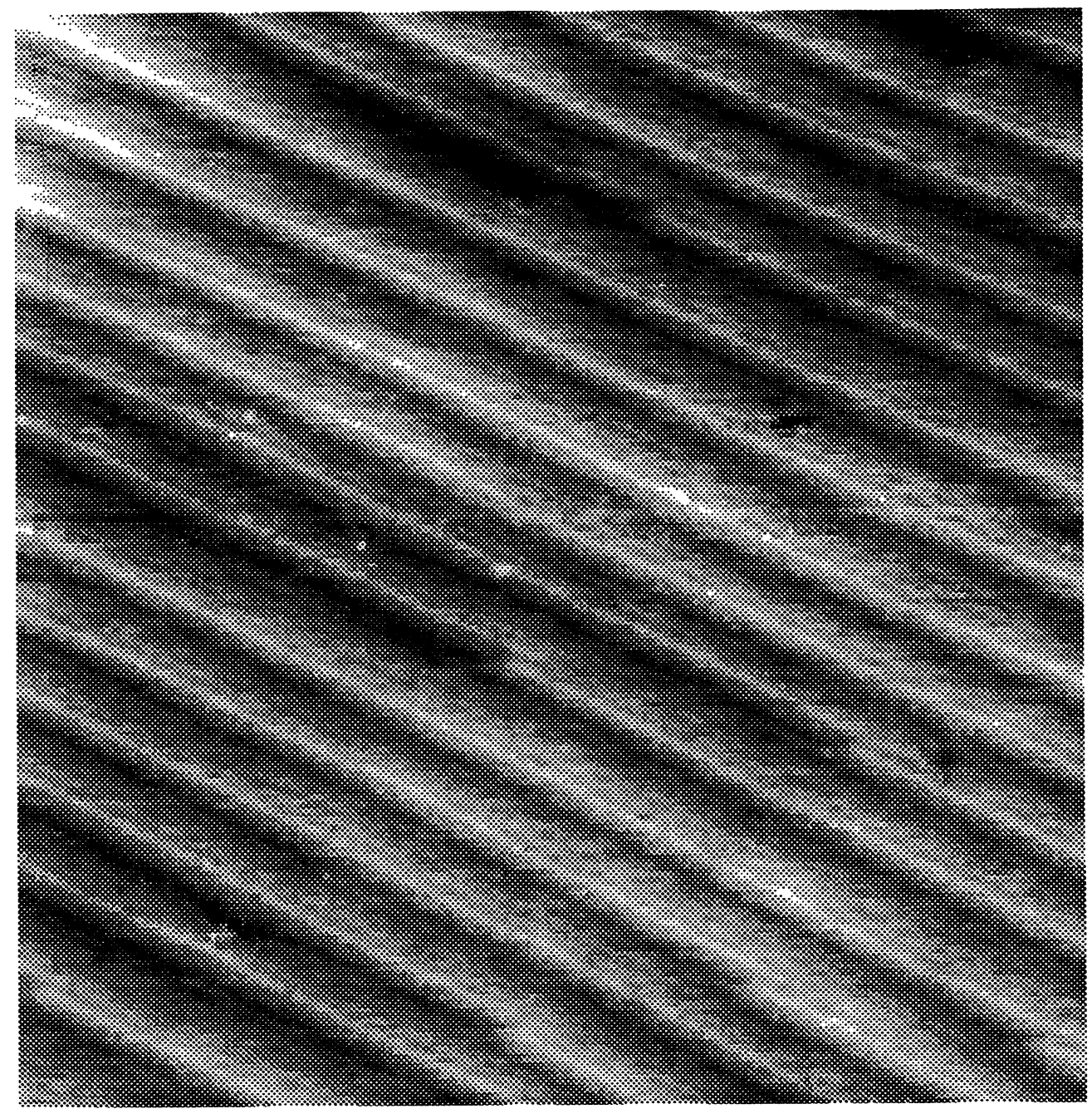

Fig. 2. - STM image of the faceted $\mathrm{Au}(4,3,3)$ surface: area $1300 \AA \times 1300 \AA$. About 17 superperiods are seen.

terraces and regular step bunches are clearly seen in figure 3. The structure of step bunches is expected to be $(7,5,5)$ orientation from previous low energy electron diffraction (LEED) studies [15]. The $(7,5,5)$ surface is also vicinal to $(1,1,1)$ in the same zone axis. Its ideal terrace width is $14.2 \AA$ (i.e. 6 atomic rows). The attractive interaction between steps separated by the distance corresponding to the period inside the step bunch is clearly demonstrated by the kink displayed in figure 3. The quantitative informations given by the STM in this study concern the value of the superperiod, $S$, the length of the $(1,1,1)$ facet, $L$, and the number $N$ of steps inside one superperiod. The value of the period inside the step bunches, $w$, can also be measured in order to confirm the $(7,5,5)$ orientation. All of these four parameters, $S, w, L$ and $N$, have been measured versus the annealing temperature. Therefore STM images have been recorded after four annealing temperatures, $720 \mathrm{~K}, 770 \mathrm{~K}, 820 \mathrm{~K}$ and $870 \mathrm{~K}$, have been used. Results are presented in figure 4 as histograms. No obvious variations of these parameters versus the temperature is seen. Therefore 


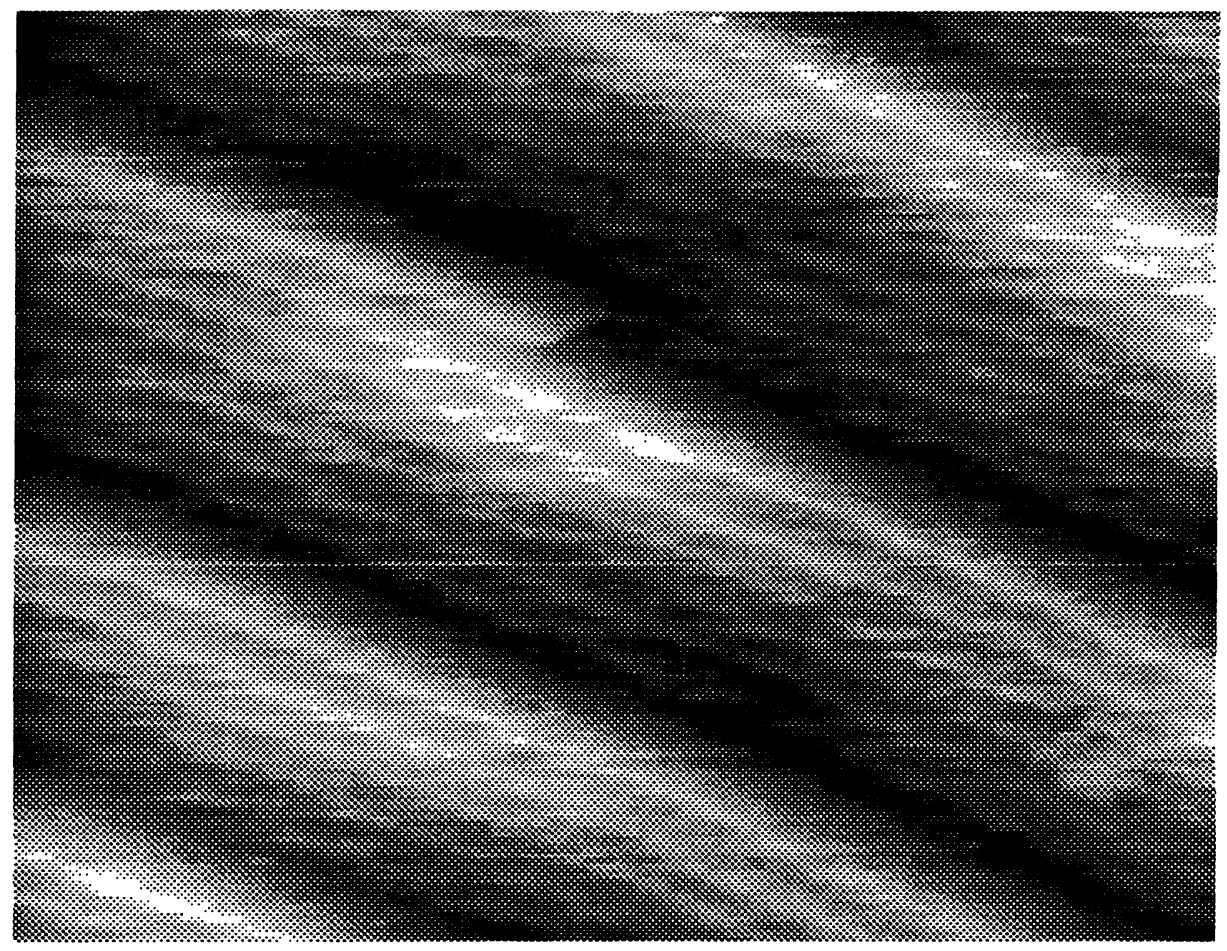

Fig. 3. - STM image of the faceted $A u(4,3,3)$ surface: area $470 \AA \times 300 \AA$. All of the steps are monoatomic, 2.35 Å high.

we believe the faceted structure does not depend on the annealing temperature in the range studied here. As a consequence, the stable state is given by the average values, $\bar{S}, \bar{w}, \bar{N}$ et $\bar{L}$ deduced from the histograms. We found that $\bar{S}=115 \AA, \bar{w}=15.6 \AA, \bar{L}=39 \AA$ and $\bar{N}=5.8$. Since STM measurements cannot be accurate better than $10 \%$, due to piezoelectric ceramics, it seems reasonable that the value found for $\bar{w}$ is consistent with the expected $(7,5,5)$ structure. Moreover the calibration of our ceramics and the average values measured here (except obviously for $\bar{N}$ ) should be corrected by the same factor $\left(\frac{14.2}{15.6}\right)$. Therefore, the value given above should be taken only as estimate rather than quantitavely. As a conclusion, the proposed model for the faceted structure is schematically represented in figure 5 . It consists in a periodic succession of $(1,1,1)$ and $(7,5,5)$ facets. The superperiod is about $110 \AA$ and the length of the $(1,1,1)$ facet is about $40 \AA$. The macroscopic orientation of the surface is checked from these average values, $\bar{N}$ and $\bar{S}$, by the relation $\tan \alpha \frac{\bar{N} \cdot h}{\bar{S}}$. This gives an angle $\alpha_{\exp }=7.4^{\circ}$ when $\bar{S}=115 \times \frac{14.2}{15.6}$. It is close to the theoretical value $\alpha_{\mathrm{th}}=8.05^{\circ}$ but not identical. Whether this discrepancy is due to measurements on STM images, to a lack of enough data and/or a real misorientation of the sample is not clear. It could also be due to other facets on the surface. However we obtained also images using a nanoscope III in air and we could check the faceted structure over areas larger than a few microns.

Finally the uncertainty on the quantitative values does not influence the qualitative results. Indeed another stepped surface, $\mathrm{Au}(11,9,9)$, in the same zone axis, has been investigated and displays qualitatively the same behaviour with a different periodicity [17]. In addition, detail LEED study on this surface appears to be consistent with the STM average value of the superperiod, about $70 \AA$ [17]. Therefore it appears as a general result that gold vicinals of $(1,1,1)$ in the [011] 
a)

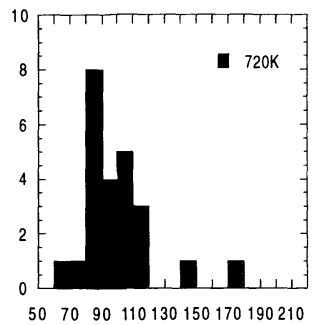

$\mathrm{S}(\mathrm{A})$

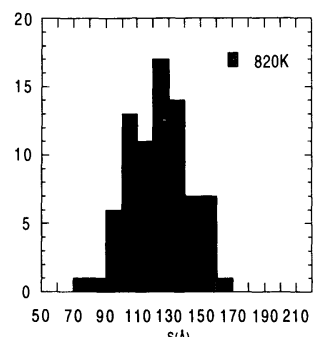

$S(A)$

c)
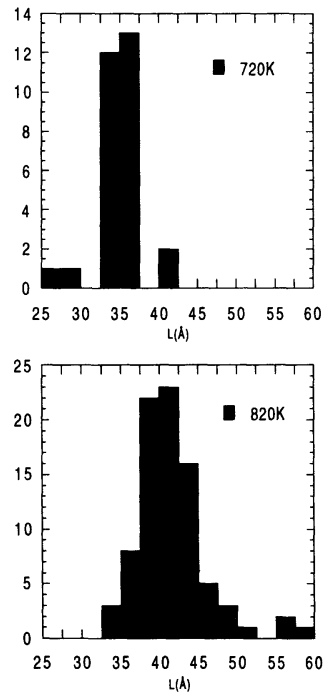

b)

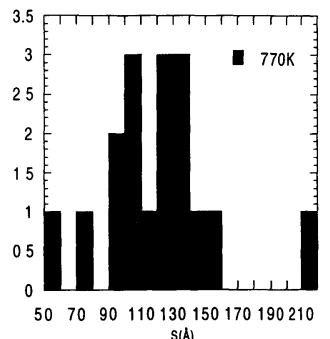

$S(A)$

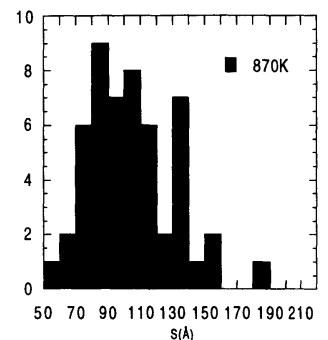

$S(A)$
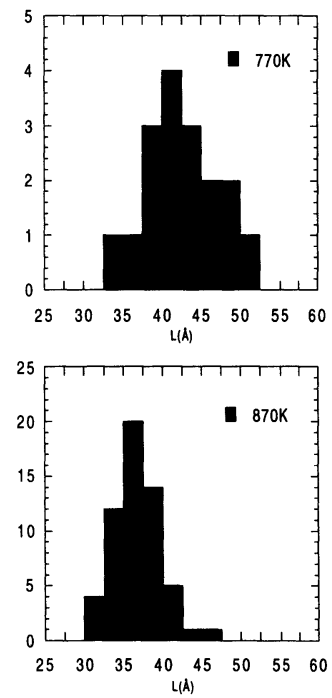

d)
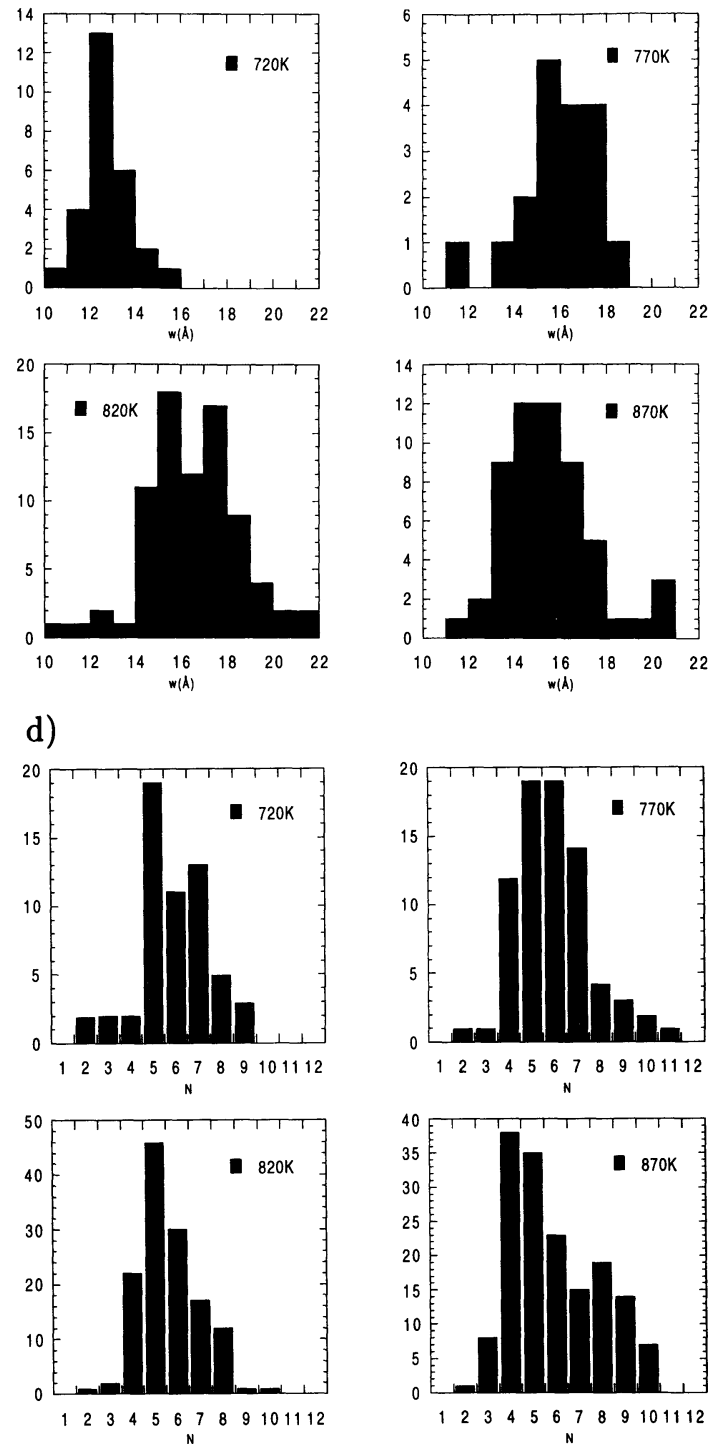

Fig. 4. - Statistical analysis of the faceted surface of $\mathrm{Au}(4,3,3)$ studied versus four annealing temperatures: $720 \mathrm{~K}, 770 \mathrm{~K}, 820 \mathrm{~K}$ and $870 \mathrm{~K}$. For each parameter $S, w, L, N, 4$ histograms are displayed, one for each temperature; a) Histograms of $S$, the superperiod. b) Histograms of $w$, the period inside step bunches. c) Histograms of $L$, the length of the (111) facet. d) Histograms of $N$, the number of steps inside one superperiod.

zone axis are unstable towards faceting and break up into a hill-and-valley structure composed of two orientations: a close-packed $(1,1,1)$ facet and a regularly stepped facet of $(7,5,5)$ orientation. In addition the surface is stable for annealing temperatures ranging from $700 \mathrm{~K}$ to $1000 \mathrm{~K}$ and a one dimensionnal periodic arrangement of the facets is clearly demonstrated using both STM and LEED. The superperiod is about $110 \AA$ for $\mathrm{Au}(4,3,3)$ and $70 \AA$ for $\mathrm{Au}(11,9,9)$. Two questions remain, which will be discussed in the following sections: $i)$ Why the occurrence of the $(7,5,5)$ structure in both cases?, ii) How can we explain the superperiod, qualitatively and quantitatively?

The question of the $(7,5,5)$ structure is first adressed. The (111) surface of gold is known to 


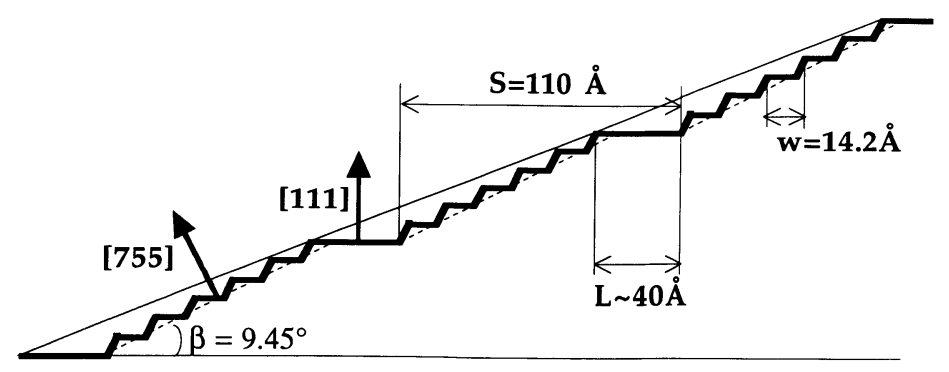

Fig. 5. - Model for the faceted $\mathrm{Au}(4,3,3)$ surface. The scale in the direction $(1,1,1)$ has been greatly enhanced for a better schematic. $S$ is the superperiod, $L$ is the length of the $(1,1,1)$ facet.

display a reconstruction. It increases the packing density of atoms in the topmost layer along the [11̄0] direction [14]. For vicinals belonging to the [011] zone axis, steps are not orthogonal to the main contraction direction giving rise to the $22 \times \sqrt{3}$ reconstruction. This is a major difference with previous investigated "magic vicinals" which makes the interpretation, here, more difficult. Moreover, steps are running along a direction that could be attributed to the largest vector of the $22 \times \sqrt{3}$ unit-cell. As a consequence, any terrace width containing an even number of rows can accomodate an integer number of unit-cell of the $22 \times \sqrt{3}$ reconstruction. Using both STM and LEED, no sign that could indicate the presence of the $22 \times \sqrt{3}$ reconstruction has been found. STM images on the faceted surfaces never display any reconstruction, although atomic resolution has been achieved on the terraces. However the $22 \times \sqrt{3}$ reconstruction can be easily imaged on a flat (111) gold surface with our microscope. Also LEED profiles do not present any spot which could arise from the $22 \times \sqrt{3}$ reconstruction although we have been able to detect closest spots due to the superperiodicity [17]. Therefore we believe that the $22 \times \sqrt{3}$ reconstruction is not present on the vicinals studied here.

The second result, we want to point out, concerns the occurrence of the $(7,5,5)$ structure. The $(7,5,5)$ plane is tilted to the (111) plane by $9.45^{\circ}$. Equilibrium shape of gold crystallites (ESC) have been observed by Heyraud and Métois [7] and present sharp edges in the vicinity of the (111) facets. The discontinuity of the tangent plane of the surface in the vicinity of the (111) facet can measured from their profiles and is about $10^{\circ}$. It is in good agreement with the tilt angle of the $(7,5,5)$ surface. Therefore since the ESC have been obtained on carefully equilibrated crystallites, this quantitative agreement suggest strongly that the periodic state of the faceted surface is indeed an equilibrium state. As a consequence, models based on spinodal decomposition can be ruled out in order to explain the superperiodicity.

It has been shown [18] that work-function variations or surface stress effects allow the surface to minimize the surface free energy by forming ordered domain structures. It is the result of the competition between the interaction energy between domains and the energy cost for domain boundaries formation. There can be several kinds of interactions between domains, such as electrostatic or elastic ones. This model has been applied to a clean reconstructed surface $\mathrm{Si}(100)$ under strain $[18,19]$ and to a chemisorbed system, $\mathrm{Cu}(110)-(2 \times 1) \mathrm{O}[18,20]$. In fact, both arguments, elastic effects or electrostatic interactions are relevant on a stepped surface. On the one hand, elastic analyses for the energy and relaxation of stepped metallic surfaces have shown that there is a large inward relaxation of the outermost atoms. The energy involved is about 0.1 $\mathrm{eV} / \AA^{2}[21]$. Therefore the $(1,1,1)$ and the $(7,5,5)$ facets are expected to present a different stress tensor. On the other hand, the work function of a metal surface varies linearly with the step density due to the Schmoluchowski effect [22]. The difference of the work functions of the $(1,1,1)$ 
and the $(7,5,5)$ orientations is of $115 \mathrm{mV}$ [15]. This model permits to qualitatively explain that the surface is unstable towards the formation of domains. However, a quantitative agreement for the superperiod is still lacking.

As a summary, using STM, a periodic faceting in the equilibrium state of the $\mathrm{Au}(4,3,3)$, vicinal of $\mathrm{Au}(1,1,1)$, has been demonstrated. For annealing temperatures ranging from $700 \mathrm{~K}$ to $1000 \mathrm{~K}$, it consists of a periodic succession of $(1,1,1)$ and $(7,5,5)$ facets. The superperiodicity is about $110 \AA$. Our results suggest that the usual $22 \times \sqrt{3}$ reconstruction cannot account directly for the stability of the $(7,5,5)$ structure. Although existing models based on work function variations or surface stress effects could account for the formation of periodic domains, theoretical work is still needed to explain quantitatively the superperiodicity.

\section{Acknowledgements.}

We gratefully acknowledge J. Lecoeur and J.-P. Bellier for providing us with the sample and its excellent preparation ex-situ. It is also a pleasure to thank J. Lecoeur for many fruitfull discussions. We want also to thank E. Lacaze who allowed us to look at our sample with a nanoscope.

\section{References}

[1] Desjonquères M.C. and Spanjaard D., Concepts in Surface Physics (Springer Verlag Berlin Heidelberg, 1993), Springer Series in Surf. Sci., R. Gomer Ed., chapter 2 and references therein.

[2] Flytzani-Stephanopoulos M. and Scmidt L.D., Prog. Surf. Phys. 9 (1979) 83.

[3] Williams E.D., Surf. Sci. 299/300 (1994) 502-524.

[4] Binnig G. and Rohrer H., Helv. Phys. Acta 55 (1982), 726; IBM J. Res. Dev. 30 (1986) 353;

Scanning Tunneling Microscopy I-III, H.-J. Güntherodt, R. Wiesendanger Eds., Springer series in Surf. Sci. 20, 28, 29 (Springer, Berlin, Heidelberg, 1992 and 1993).

[5] Williams E.D., Phaneuf R.J., Wei J., Bartelt N.C. and Einstein T.L., Surf. Sci. 294 (1993) 219-242.

[6] Hahn E., Schief H., Marsico V., Fricke A. and Kern K., Phys. Rev. Lett. 72 (1994) 3378;

Ozcomert J., Pai W., Bartelt N. and Reutt-Robey J., Phys. Rev. Lett. 72 (1994), 258;

Zuo J.K., Warmack R.J., Zehner D.M. and Wendelken J.F., Phys. Rev. B 47 (1993) 10743;

Zuo J.K., Zehner D.M., Wendelken J.F., Warmack R.J. and Yang N.H., Surf. Sci. 301 (1994) 233.

[7] Heyraud J.C. and Métois J.J., Surf. Sci. 128 (1983) 334; Surf. Sci. 177 (1986) 213;

Wortis M., in Chemistry and Physics of Solid Surfaces VII, R. Vanselow and R. Howe Eds. (Springer Verlag, Berlin, 1988);

Heyraud J.C., thesis 1987, University of Aix-Marseille, unpublished.

[8] Breuer U. and Bonzel H.P., Surf. Sci. 273 (1992) 219.

[9] Herring C., Phys. Rev. 82 (1951) 87.

[10] Sotto M. and Boulliard J.C., Surf. Sci. 214 (1988) 97;

Samson Y., Rousset S., Gauthier S., Girard J.C. and Klein J., Surf. Sci. 315 (1994) L969.

[11] Kaiser W.J. and Jaklevic R.C., Surf. Sci. 182 (1987) L227.

[12] Bartolini A., Ercolessi F. and Tosatti E., Phys. Rev. Lett. 63 (1989) 872.

[13] Yamazaki K., Takayanagi K., Tanishiro Y. and Yagi K., Surf. Sci. 199595 (1988) and references therein.

[14] Harten U., Lahee A.M., Peter Toennies J. and Wöll Ch., Phys. Rev. Lett. 54 (1985) 2619;

Wöll Ch., Chiang S., Wilson R.J. and Lippel P.H., Phys. Rev. B39 (1989) 11973 and references therein.

[15] Lecoeur J., Bellier J.P. and Koehler C., J. Electroanal. Chem. 337 (1992) 197;

Lecoeur J., Bellier J.P. and Koehler C., J. Electroanal. Chem. (1994) in press.

[16] Siboulet O., thesis 1991, University of Paris 7, unpublished.

[17] Pourmir F., Rousset S., Gauthier S., Sotto M., Klein J., Lecoeur J. and Bellier J.P. (to be published in Surf. Sci. Lett.).

[18] Alerhand O.L., Vanderbilt D., Meade R.D. and Joannopoulos J.D., Phys. Rev. Lett. 61 (1988) 1973; 
Vanderbilt D., Surf. Sci. Lett. 268 (1992) L300-L304.

[19] Men F.K., Packard W.E. and Webb M.B., Bull. Am. Phys. Soc. 33 (1988) 472; Phys. Rev. Lett. 61 (1988) 2469.

[20] Kern K., Niehus H., Schatz A., Zeppenfeld P., . George J. and Comsa G., Phys. Rev. Lett. 67 (1991) 855.

[21] Srolovitz D.J. and Hirth J.P., Surf. Sci. 255 (1991) 111-119.

[22] Schmoluchowski R., Phys. Rev. 60 (1941) 661;

Besocke K., Krahl-Urban B., Wagner H., Surf. Sci. 68 (1977) 39;

Wagner H. in Solid Surface Physics, Springer Tracts in Modern Physics, Vol. 85 (Springer Verlag). 